\title{
Isolation, Morphological and Cultural Characterization of Azospirillum Isolated from Rhizospheric Soils of Various Non-Leguminous Crops of Ranchi Having Acidic pH
}

\author{
R. Narayan", N.C. Gupta and D.K. Shahi \\ Department of Soil Science and Agricultural Chemistry, Birsa Agricultural University, \\ Ranchi, Jharkhand, India \\ *Corresponding author
}

\begin{abstract}
A B S T R A C T
Azospirillum is one of the versatile non-symbiotic, free living diazotrophic bacteria which appears to have a world-wide distribution and occurs in large number in the rhizosphere

Keywords

Azospirillum,

Rhizosphere,

Diazotrophic bacteria,

Isolation,

Characterisation,

Microcyst, Capsule soil of a variety of grasses and cereals. The present study was carried out during Rabi and Kharif 2016-17 in the Department of Soil Science and Agricultural Chemistry, Birsa Agricultural University, Ranchi, Jharkhand. Efforts were made to screen out the presence of Azospirillum in rhizosphere of various non-leguminous crops and to characterize the isolates on the basis of morphological and cultural behaviours. On the basis of $\mathrm{pH}$ range (4.0-5.5), 54 rhizospheric soil samples were tentatively selected out of 100 samples for investigation. From the study conducted, presence of Azospirillum in rhizosphere of acidic

Article Info

Accepted:

04 July 2018

Available Online:

10 August 2018 $\mathrm{pH}$ was confirmed. Morphological characterization revealed that Azospirillum isolated from rhizosphere of various crops were gram negative and vibroid in shape. Cells were encapsulated i.e., were having capsules around them and formed microcyst in aged culture. Cultural characterisation revealed that colonies developed on agar slants were smooth, some of them were having raised while others were having flat elevation. Amount of growth of colonies observed were dense in 43 and thin in 11 colonies while they developed white sub-surface pellicle when grown in semi-solid Okon's media. Out of 54 colonies, 41 were white, 5 were red and rest colonies were found yellow in colour.
\end{abstract}

\section{Introduction}

Rhizosphere soil is a "hot-spot" for microbial growth and major microbial activities (Sachdev et al., 2009). It is the narrow zone of soil specifically influenced by the root system (Dobbelaere et al., 2003). This zone is rich in nutrients when compared with the bulk soil due to the accumulation of a variety of plant exudates such as amino acids and sugars providing a rich source of energy and nutrients for bacteria (Gray and Smith 2005). Root exudates are the substrate or fuel for the intense microbial (bacteria, fungi, algae, protozoa, nematodes and arthropods) activity within the rhizosphere. Thus it is the quantity and quality of the exudates and condition of the soil habitat that will determine the colonization potential of the rhizosphere (Lugtenberg et al., 2002). Azospirillum spp. isolated from various geographical regions of the world is one of the best-characterized 
genus of plant growth-promoting rhizobacteria (PGPR). They are known to associate with the roots of wheat, tropical grasses, maize, and other cereals (Oh et al., 1999). The soil bacterium Azospirillum was first isolated from the Netherlands and originally named as Spirillum lipoferum by Beijerinck et al., (1925). Later Schroder (1932) isolated from the soils in Germany and Austria. Till now, they have been isolated from the rhizosphere of many grasses and cereals all over the world, in a wide variety of terrestrial and aquatic habitats of tropical as well as in temperate climates (Yooshinan, 2001). Its occurrence in the rhizosphere varied from 1 to 10 per cent to the total rhizosphere population (Okon, 1985). Azospirilla are gram-negative, free-living, nitrogen-fixing rhizospheric bacteria.

They display a versatile $\mathrm{C}$ and $\mathrm{N}$ metabolism which makes them well adapted to establish in the competitive environment of the rhizosphere (Hartmann and Zimmer 1994). Azospirillum flocs comprise a mixture of vegetative and encysted cells surrounded by a polysaccharide-rich network (capsule), conferring advantages such as stress tolerance, extended shelf life and enhanced survivability (Sadasivan and Neyra, 1985). Azospirillum cells appear in two distinct forms: the slightly vibroid form (V-form) occurring in young laboratory cultures and on plant roots (Tarrand et al., 1978), and the cyst form (C-form), occurring under stress or in old laboratory cultures (Sadasivan and Neyra, 1985). The Cform may be a survival structure. Occurrence of Azospirillum in soil is strongly $\mathrm{pH}-$ dependent with a $\mathrm{pH}$ around 7 , being optimal. However, sporadic occurrence was observed even in soils with $\mathrm{pH} 4.8$ (Magalhaes et al., 1983). Hence the present work was undertaken with a view to screen out the presence, isolate Azospirillum spp. from the rhizospheres of acidic soils of Ranchi (Jharkhand) and characterise them on the basis of their morphological and cultural behaviour.

\section{Materials and Methods}

\section{Material}

Azospirillum species studied in the present investigation were isolated from soil of rhizosphere having $\mathrm{pH}$ range of 4.0 to 5.5 of different non-leguminous crops grown in various blocks viz., Kanke, Aangara, Nagri, Bero, Itki of Ranchi district. Details of the location, soil $\mathrm{pH}$ and crop grown selected for isolation of Azospirillum are mentioned in Table 1.

\section{Collection of rhizosphere soil}

Rhizosphere soils were collected from the rhizospheric region of the plant at the depth of 5-6 cm near the periphery of roots of different crops from different blocks of Ranchi district in plastic bags. The soil samples were preserved in refrigerator.

\section{pH of soil samples}

Soil samples were collected from 100 different locations from Ranchi districts for $\mathrm{pH}$ analysis. The soil samples were air dried, grounded, sieved for estimation of $\mathrm{pH}$ by adopting standard methods. Soil $\mathrm{pH}$ was determined in a soil water suspension of 1:2.5 $\mathrm{w} / \mathrm{v}$, stirred at regular intervals for 30 minutes using pH meter (Jackson 1973). Details of selected 54 soil samples selected for isolation of Azospirillum has been presented in Table 1.

\section{Isolation of Azospirillum spp.}

Isolation of Azospirillum species from rhizospheric soils was done following the methods of serial dilution. From the soil samples selected on the basis of $\mathrm{pH}$ range (4.0-5.5), $1 \mathrm{~g}$ of soil was taken and serially diluted using sterile distilled water upto $10^{-6}$ dilutions. One $\mathrm{ml}$ of diluted sample from $10^{-4}$ to $10^{-6}$ dilutions were taken and $1 \mathrm{ml}$ of aliquot 
was inoculated in tubes containing Okon's $\mathrm{Nfb}$ (Nitrogen free bromothymol) semi-solid media. All the tubes were incubated at $35^{\circ} \mathrm{C}$ for $48 \mathrm{~h}$ and observed the growth by the formation of pellicles. Pellicles formation is considered as positive for Azospirillum.

Pellicles were streaked on petriplates containing $\mathrm{Nfb}$ Okon's solid media and incubated at $35^{\circ} \mathrm{C}$ for 48 hours. Morphologically divergent Azospirillum colonies were picked from the plates of dilution $10^{-5}$ and streaked on basal minimal salt agar medium and incubated at $35^{\circ} \mathrm{C}$ for 24-48 hrs.

After attaining sufficient growth, all the isolates were preserved in a refrigerator for further investigation. The colonies developed on Okon's agar medium ( $\mathrm{pH}$ adjusted to 6.8) were transferred to slants of same medium and stored at $4^{0} \mathrm{C}$.

\section{Okon's Media}

Malic acid $5.00 \mathrm{~g}, \mathrm{KOH} 4.00 \mathrm{~g}, \mathrm{~K}_{2} \mathrm{HPO}_{4} 0.50$ g, FeSO4.7H2O $0.05 \mathrm{~g}, \mathrm{MnSO}_{4} .7 \mathrm{H}_{2} \mathrm{O} 0.01 \mathrm{~g}$, $\mathrm{MgSO}_{4} .7 \mathrm{H}_{2} \mathrm{O} \quad 0.10 \mathrm{~g}, \mathrm{NaCl} 0.02 \mathrm{~g}, \mathrm{CaCl}_{2}$ $0.01 \mathrm{~g}, \mathrm{Na}_{2} \mathrm{MoO}_{4} 0.002 \mathrm{~g}$, Bromothymol blue (0.5\% in $95 \%$ methanol) $2.00 \mathrm{ml}$, Agar $1.8 \mathrm{~g}$ (semi-solid)/18 g(solid), $\mathrm{NH}_{4} \mathrm{Cl} 1 \mathrm{~g}$, Water 1 litre.

\section{Purification of the culture}

Purification of the culture was carried out by frequent transfer of colony of Azospirillum developed on Okon's agar media to seal solid nitrogen free malate medium on petriplates (Okon et al., 1977) having the following constituents: $\mathrm{K}_{2} \mathrm{HPO}_{4} 6.0 \mathrm{~g}, \mathrm{KH}_{2} \mathrm{PO}_{4} 4.0 \mathrm{~g}$, $\mathrm{MgSO}_{4} 7 \mathrm{H}_{2} \mathrm{O} 0.2 \mathrm{~g}, \mathrm{NaCl} 0.1 \mathrm{~g}, \mathrm{CaCl}_{2} 0.2 \mathrm{~g}$, $\mathrm{NH}_{4} \mathrm{Cl} 0.1 \mathrm{~g}, \mathrm{NaOH} 3.0 \mathrm{~g}$, Yeast extract $0.1 \mathrm{~g}$, $\mathrm{FeCl}_{3} 10.0 \mathrm{mg}, \mathrm{Na}_{2} \mathrm{MoO}_{4} 20.00 \mathrm{mg}, \mathrm{MnSO}_{4}$ $2.10 \mathrm{mg}, \mathrm{H}_{3} \mathrm{BO}_{3} 2.80 \mathrm{mg}, \mathrm{Cu}\left(\mathrm{NO}_{3}\right)_{2} 0.04 \mathrm{mg}$, Agar $18 \mathrm{~g}$, Water 1 litre

\section{Morphological characterization}

\section{Gram reaction}

Smears prepared from 48 hours old cultures were gram stained as per Huker modification (Rangaswami and Bagyaraj, 1996).

The slides were observed under compound microscope (oil immersion).

\section{Capsule staining}

Presence of capsules around the cells was observed on acetic crystal violet stained smears under oil immersion.

\section{Microcyst formation}

Stained smears of two weeks old cultures were observed under oil immersion.

Observations were recorded regarding presence of round thick walled cells as the preparation of microcysts.

\section{Shape}

Smears prepared from 48 hours old cultures were obtained and examined under oil immersion.

\section{Cultural characterization}

Different isolates of Azospirillum species were grown on respective standard media and their characteristic growth patterns were observed.

Serially diluted isolates of Azospirillum species were grown on Okon's agar medium (Okon et al., 1977) in petriplates and in tubes (for agar strokes) at $35^{\circ} \mathrm{C}$ for 72 hours then purification of colonies were done.

Observations were made with regard to nature of colonial growth. 


\section{Results and Discussion}

In the present study, selectivity to grow on specific $\mathrm{Nfb}$ (Nitrogen free bromothymol) media and subsequently confirming their morphological, cultural and physiological identity with the type cultures as described in Bergey's Manual (Buchanan and Gibbons, 1974) and Aquaspirillum taxonomy for Spirillum (Kreig and Hylemon, 1976) were taken as reference for investigation and characterization of Azospirillum isolates. A total of 54 isolates were studied under various morphological and cultural behaviours.

\section{Morphological characteristics}

All the isolates were studied for their morphological characteristics and results are presented in Table 2. Isolates were microscopically observed for their gram reaction, cell shape, presence of capsule and microcyst formation. Results revealed that the 54 isolates were gram negative in reaction and cell shape of all the isolates was vibroid when observed under microscope. These findings were confirmed by Rosemary et al., 2013 and Rasool et al., 2015. All the isolates were having capability of forming microcysts. Transition into cyst-like cells were observed in older cultures of Azospirillum was reported by Berlman (2004). Extracellular capsule was present in all 54 isolates which is in confirmity with reports of Madi et al., (1988).

\section{Cultural characteristics}

Data related to cultural characterisation has been presented in Table 2 .

\section{Colony morphology}

Study revealed that colonies developed on agar slants were smooth, some of them were having raised while others were having flat elevation. Amount of growth ranged from large to slight. 43 colonies were dense and 11 were thin in amount of growth.

Azospirillum displays high degree of pleomorphism with cellular and colony variations among the species as well as within each species depending on the strain, medium composition and culture conditions as reported by Becking, 1985 . The same was investigated by Rasool et al., (2015).

Fig.1 White colonies of Azospirillum

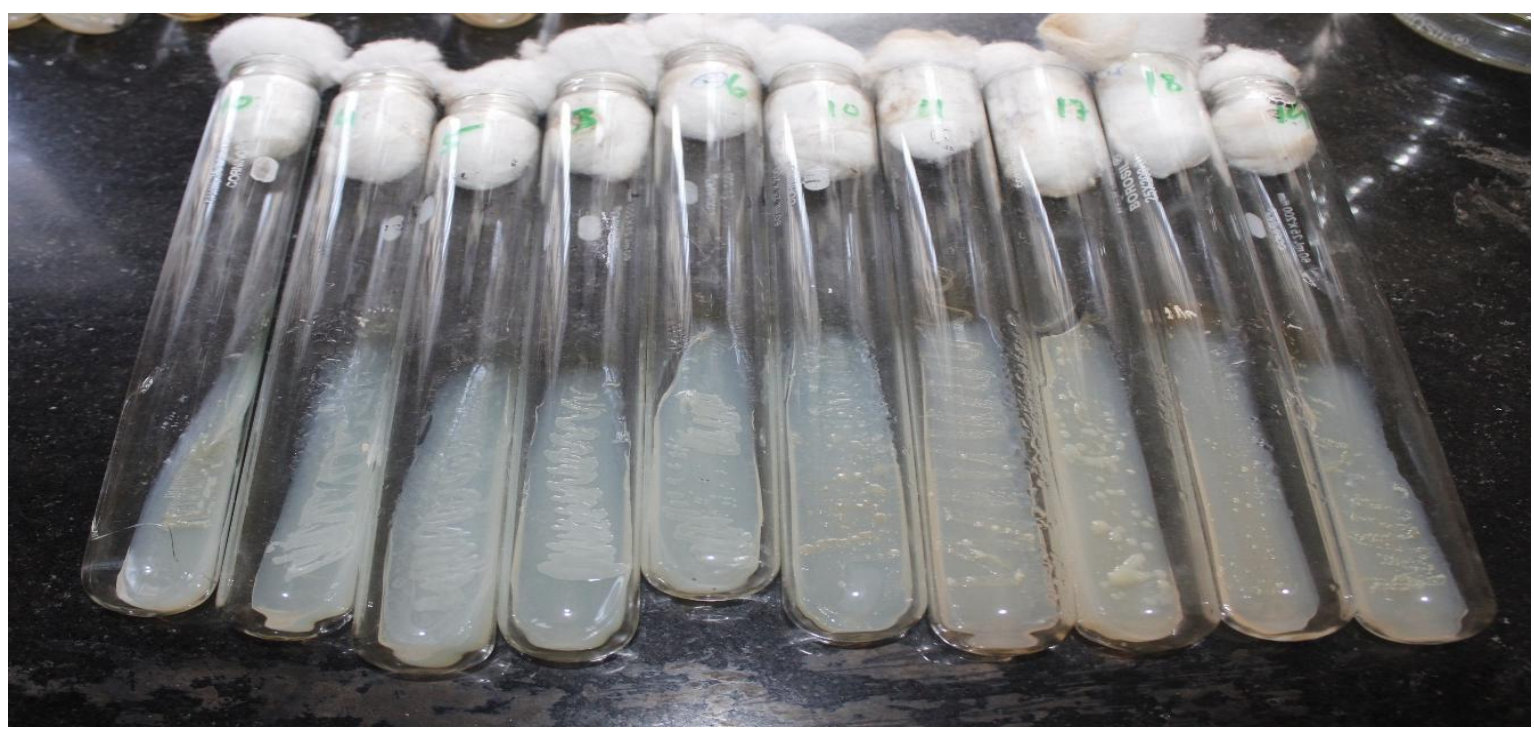


Int.J.Curr.Microbiol.App.Sci (2018) 7(8): 329-338

Fig.2 Yellow colonies of Azospirillum

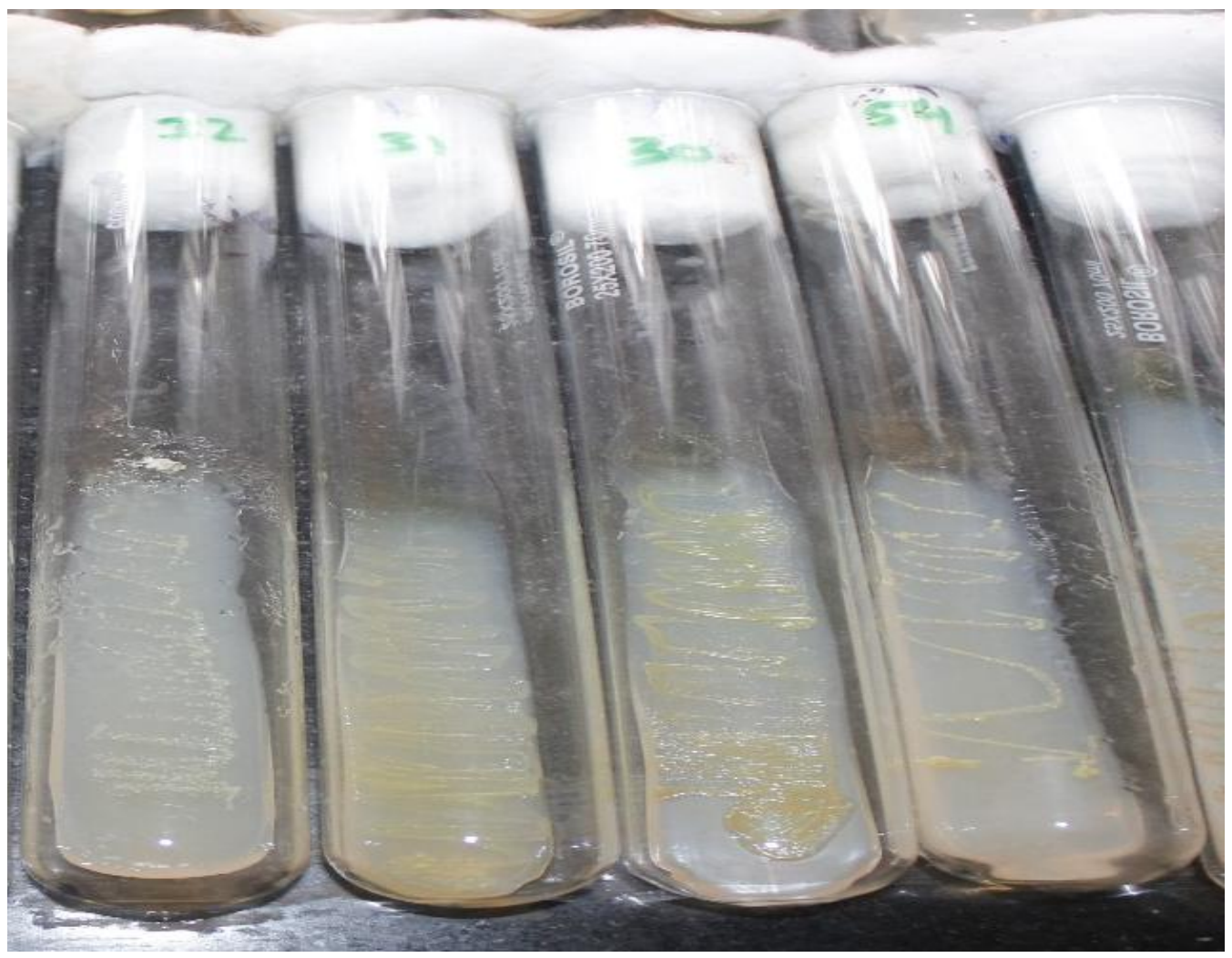

Fig.3 Red colonies of Azospirillum

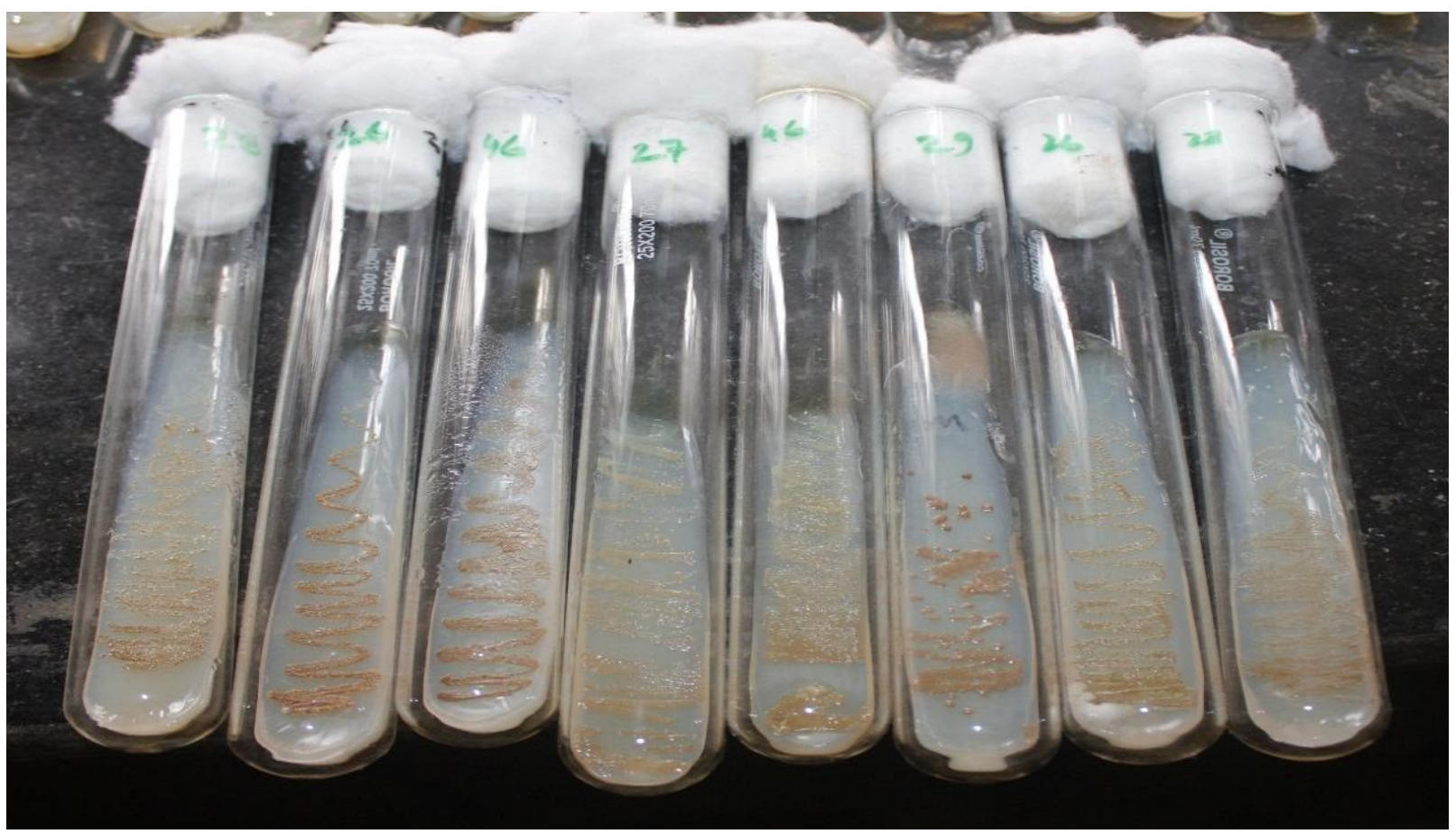


Table.1 Details of 54 rhizospheric soil samples selected for isolation of Azospirillum

\begin{tabular}{|c|c|c|c|c|}
\hline Sl. No. & Sample. No. & Place of collection & $\begin{array}{c}\text { pH of the } \\
\text { soil }\end{array}$ & Crop (previous/ present) \\
\hline 1 & AZM5 & B.A.U Campus, SSAC, Kanke block & 5.4 & Maize \\
\hline 2 & AZM6 & B.A.U Campus, SSAC, Kanke block & 5.3 & Maize \\
\hline 3 & AZM10 & B.A.U Campus, SSAC, Kanke block & 5.5 & Rice \\
\hline 4 & AZM15 & B.A.U Campus, Tech park,Kanke block & 5.1 & Rice \\
\hline 5 & AZM16 & B.A.U Campus, Tech park, Kanke block & 5.4 & Ragi \\
\hline 6 & AZM17 & R.A.C Farm, W-section, Kanke block & 5.3 & Rice \\
\hline 7 & AZM18 & R.A.C Farm, W-section, Kanke block & 5.1 & Ragi \\
\hline 8 & AZM19 & R.A.C Farm, W-section, Kanke block & 5.2 & Rice \\
\hline 9. & AZM22 & R.A.C Farm, W-section, Kanke block & 5.5 & Wheat \\
\hline 10. & AZM23 & R.A.C Farm, W-section, Kanke block & 5.4 & Wheat \\
\hline 11. & AZM25 & Chamghati, Aangara block & 5.5 & Rice \\
\hline 12. & AZM26 & Chamghati, Aangara block & 5.3 & Rice \\
\hline 13. & AZM27 & Chamghati, Aangara block & 5.1 & Rice \\
\hline 14. & AZM29 & Chamghati, Aangara block & 5.2 & Rice \\
\hline 15. & AZM30 & Chamghati, Aangara block & 5.4 & Rice \\
\hline 16. & AZM32 & Chamghati, Aangara block & 5.4 & Rice \\
\hline 17. & AZM33 & Chamghati, Aangara block & 5.3 & Rice \\
\hline 18. & AZM34 & Chamghati, Aangara block & 5.2 & Rice \\
\hline 19. & AZM35 & Chauli patra, Nagri block & 4.9 & Pea \\
\hline 20. & AZM36 & Chauli patra, Nagri block & 4.6 & Ragi \\
\hline 21. & AZM 39 & Itki mor, Itki block & 4.7 & Potato \\
\hline 22. & AZM 40 & Itki mor, Itki block & 4.6 & Ragi \\
\hline 23. & AZM 42 & Itki mor, Itki block & 4.7 & Mustard + Pea \\
\hline 24. & AZM 45 & Garhgao, Itki block & 4.6 & Pea + Sugarcane \\
\hline 25. & AZM 46 & Garhgao, Itki block & 5.1 & Wheat \\
\hline 26. & AZM 53 & Devali, Itki block & 5.4 & Ragi \\
\hline 27. & AZM 55 & Devali, Itki block & 4.7 & Potato \\
\hline 28. & AZM 56 & Devali, Itki block & 4.8 & Maize \\
\hline 29. & AZM 60 & Bhandra, Itki block & 4.2 & Maize \\
\hline 30. & AZM 61 & Bhandra, Itki block & 4.7 & Onion \\
\hline 31 & AZM 62 & Karmatoli, Bero block & 4.1 & Pea + Potato \\
\hline 32. & AZM 63 & Karmatoli, Bero block & 4.0 & Potato \\
\hline 33. & AZM 64 & Karmatoli, Bero block & 4.0 & Potato \\
\hline 34. & AZM 65 & Kalanji, Bero block & 4.0 & Ginger \\
\hline 35. & AZM 66 & Didhiya, Bero block & 4.1 & Mustard + Pea \\
\hline 36. & AZM 70 & Tuko, Bero block & 5.1 & Pea \\
\hline 37. & AZM 71 & Tuko, Bero block & 4.4 & Potato \\
\hline 38. & AZM 75 & Parepara, Bero block & 4.6 & Pea \\
\hline 39. & AZM 76 & Parepara, Bero block & 4.9 & Potato \\
\hline 40. & AZM 77 & Parepara, Bero block & 4.7 & Lentil \\
\hline 41. & AZM 80 & Jainathpur, Bero block & 4.8 & Pea \\
\hline 42. & AZM 81 & Jainathpur, Bero block & 4.9 & Mustard \\
\hline 43. & AZM 83 & Bhaishmuro, Bero block & 4.4 & Ginger \\
\hline 44. & AZM 84 & Bhaishmuro, Bero block & 4.8 & Mustard \\
\hline 45. & AZM 85 & Bhaishmuro, Bero block & 4.4 & Pea \\
\hline 46. & AZM 87 & Bhaishmuro, Bero block & 4.1 & Ragi \\
\hline 47. & AZM 88 & Bhaishmuro, Bero block & 4.3 & Potato \\
\hline 48. & AZM 89 & Bhaishmuro, Bero block & 4.4 & Potato \\
\hline 49. & AZM 90 & Bhaishmuro, Bero block & 4.1 & Potato \\
\hline 50. & AZM 93 & Kundo, Bero block & 4.2 & Potato \\
\hline 51. & AZM 94 & Kundo, Bero block & 4.8 & Ragi \\
\hline 52. & AZM 95 & Kundo, Bero block & 4.6 & Maize \\
\hline 53. & AZM 99 & Bero, Bero block & 4.3 & Potato \\
\hline 54. & AZM 100 & Bero, Bero block & 4.7 & Ragi \\
\hline
\end{tabular}


Table.2 Morphological and cultural characterization of the new isolates of Azospirillum

\begin{tabular}{|c|c|c|c|c|c|c|c|c|}
\hline $\begin{array}{l}\text { Sl. } \\
\text { No. }\end{array}$ & $\begin{array}{c}\text { Azospirillum } \\
\text { isolates }\end{array}$ & $\begin{array}{c}\text { Gram } \\
\text { reaction }\end{array}$ & Capsule & $\begin{array}{l}\text { Microcyst } \\
\text { formation }\end{array}$ & $\begin{array}{c}\text { Shape of } \\
\text { cell }\end{array}$ & Solid agar media & Semi-solid media & $\begin{array}{l}\text { Color of } \\
\text { colony }\end{array}$ \\
\hline 1. & AZM 5 & Negative & Present & + & Vibroid & Smooth, Raised, Dense & White sub-surface pellicle & White \\
\hline 2. & AZM 6 & Negative & Present & + & Vibroid & Smooth, Raised, Dense & White sub-surface pellicle & White \\
\hline 3. & AZM 10 & Negative & Present & + & Vibroid & Smooth, Flat, Dense & White sub-surface pellicle & White \\
\hline 4. & AZM 15 & Negative & Present & + & Vibroid & Smooth, Flat, Dense & White sub-surface pellicle & White \\
\hline 5. & AZM 16 & Negative & Present & + & Vibroid & Smooth, Flat, Dense & White sub-surface pellicle & White \\
\hline 6. & AZM 17 & Negative & Present & + & Vibroid & Smooth, Flat, Dense & White sub-surface pellicle & White \\
\hline 7. & AZM 18 & Negative & Present & + & Vibroid & Smooth, Flat, Dense & White sub-surface pellicle & White \\
\hline 8. & AZM 19 & Negative & Present & + & Vibroid & Smooth, Flat, Dense & White sub-surface pellicle & White \\
\hline 9. & AZM 22 & Negative & Present & + & Vibroid & Smooth, Raised, Dense & White sub-surface pellicle & White \\
\hline 10. & AZM 23 & Negative & Present & + & Vibroid & Smooth, Raised, Dense & White sub-surface pellicle & White \\
\hline 11. & AZM 25 & Negative & Present & + & Vibroid & Smooth, Flat, Dense & White sub-surface pellicle & White \\
\hline 12. & AZM 26 & Negative & Present & + & Vibroid & Smooth, Raised, Dense & White sub-surface pellicle & White \\
\hline 13. & AZM 27 & Negative & Present & + & Vibroid & Smooth, Raised, Dense & White sub-surface pellicle & White \\
\hline 14. & AZM 29 & Negative & Present & + & Vibroid & Smooth, Raised, Dense & White sub-surface pellicle & White \\
\hline 15. & AZM 30 & Negative & Present & + & Vibroid & Smooth, Flat, Dense & White sub-surface pellicle & White \\
\hline 16. & AZM 32 & Negative & Present & + & Vibroid & Smooth, Flat, Dense & White sub-surface pellicle & White \\
\hline 17. & AZM 33 & Negative & Present & + & Vibroid & Smooth, Flat, Dense & White sub-surface pellicle & White \\
\hline 18. & AZM 34 & Negative & Present & + & Vibroid & Smooth, Flat, Dense & White sub-surface pellicle & White \\
\hline 19. & AZM 35 & Negative & Present & + & Vibroid & Smooth, Flat, Dense & White sub-surface pellicle & White \\
\hline 20. & AZM 36 & Negative & Present & + & Vibroid & Smooth, Flat, Dense & White sub-surface pellicle & White \\
\hline 21. & AZM 39 & Negative & Present & + & Vibroid & Smooth, Flat, Dense & White sub-surface pellicle & White \\
\hline 22. & AZM 40 & Negative & Present & + & Vibroid & Smooth, Flat, Dense & White sub-surface pellicle & White \\
\hline 23. & AZM 42 & Negative & Present & + & Vibroid & Smooth, Flat, Dense & White sub-surface pellicle & White \\
\hline 24. & AZM 45 & Negative & Present & + & Vibroid & Smooth, Flat, Dense & White sub-surface pellicle & White \\
\hline 25. & AZM 46 & Negative & Present & + & Vibroid & Smooth, Flat, Dense & White sub-surface pellicle & White \\
\hline
\end{tabular}




\begin{tabular}{|c|c|c|c|c|c|c|c|c|}
\hline 26. & AZM 53 & Negative & Present & + & Vibroid & Smooth, Flat, Dense & White sub-surface pellicle & White \\
\hline 27. & AZM 55 & Negative & Present & + & Vibroid & Smooth, Flat, Dense & White sub-surface pellicle & White \\
\hline 28. & AZM 56 & Negative & Present & + & Vibroid & Smooth, Flat, Dense & White sub-surface pellicle & White \\
\hline 29. & AZM 60 & Negative & Present & + & Vibroid & Smooth, Flat, Dense & White sub-surface pellicle & White \\
\hline 30. & AZM 61 & Negative & Present & + & Vibroid & Smooth, Flat, Dense & White sub-surface pellicle & White \\
\hline 31. & AZM 62 & Negative & Present & + & Vibroid & Smooth, Flat, Thin & White sub-surface pellicle & Red \\
\hline 32. & AZM 63 & Negative & Present & + & Vibroid & Smooth, Flat, Thin & White sub-surface pellicle & Red \\
\hline 33. & AZM 64 & Negative & Present & + & Vibroid & Smooth, Flat, Thin & White sub-surface pellicle & Red \\
\hline 34. & AZM 65 & Negative & Present & + & Vibroid & Smooth, Flat, Thin & White sub-surface pellicle & Red \\
\hline 35. & AZM 66 & Negative & Present & + & Vibroid & Smooth, Flat, Thin & White sub-surface pellicle & Yellow \\
\hline 36. & AZM 70 & Negative & Present & + & Vibroid & Smooth, Flat, Thin & White sub-surface pellicle & Yellow \\
\hline 37. & AZM 71 & Negative & Present & + & Vibroid & Smooth, Flat, Thin & White sub-surface pellicle & Yellow \\
\hline 38. & AZM 75 & Negative & Present & + & Vibroid & Smooth, Flat, Dense & White sub-surface pellicle & White \\
\hline 39. & AZM 76 & Negative & Present & + & Vibroid & Smooth, Flat, Dense & White sub-surface pellicle & White \\
\hline 40. & AZM 77 & Negative & Present & + & Vibroid & Smooth, Flat, Dense & White sub-surface pellicle & White \\
\hline 41. & AZM 80 & Negative & Present & + & Vibroid & Smooth, Flat, Dense & White sub-surface pellicle & Yellow \\
\hline 42. & AZM 81 & Negative & Present & + & Vibroid & Smooth, Flat, Dense & White sub-surface pellicle & Yellow \\
\hline 43. & AZM 83 & Negative & Present & + & Vibroid & Smooth, Flat, Thin & White sub-surface pellicle & White \\
\hline 44. & AZM 84 & Negative & Present & + & Vibroid & Smooth, Flat, Thin & White sub-surface pellicle & White \\
\hline 45. & AZM 85 & Negative & Present & + & Vibroid & Smooth, Flat, Thin & White sub-surface pellicle & White \\
\hline 46. & AZM 87 & Negative & Present & + & Vibroid & Smooth, Flat, Thin & White sub-surface pellicle & Red \\
\hline 47. & AZM 88 & Negative & Present & + & Vibroid & Smooth, Flat, Dense & White sub-surface pellicle & White \\
\hline 48. & AZM 89 & Negative & Present & + & Vibroid & Smooth, Flat, Dense & White sub-surface pellicle & White \\
\hline 49. & AZM 90 & Negative & Present & + & Vibroid & Smooth, Flat, Dense & White sub-surface pellicle & White \\
\hline 50. & AZM 93 & Negative & Present & + & Vibroid & Smooth, Flat, Dense & White sub-surface pellicle & White \\
\hline 51. & AZM 94 & Negative & Present & + & Vibroid & Smooth, Flat, Dense & White sub-surface pellicle & White \\
\hline 52. & AZM 95 & Negative & Present & + & Vibroid & Smooth, Flat, Dense & White sub-surface pellicle & Yellow \\
\hline 53. & AZM 99 & Negative & Present & + & Vibroid & Smooth, Flat, Dense & White sub-surface pellicle & Yellow \\
\hline 54. & AZM 100 & Negative & Present & + & Vibroid & Smooth, Flat, Dense & White sub-surface pellicle & Yellow \\
\hline
\end{tabular}




\section{Colour production by colonies}

Out of 54 colonies, colour of 41 was white, 5 were red and rest were yellow in colour (Fig. 1, 2 and 3). Tarrand et al., (1978) have reported that colonies of different $\mathrm{N}_{2}$ fixing Azospirillum strain showed pink, deep pink, red or yellow colour. This was due to presence of different carotenoid pigment in that isolates as reported by Baldani et al., (1986) and Rasool et al., (2015).

\section{Growth in semi-solid media}

Investigation revealed that all the 54 isolates of Azospirillum were developed as white subsurface pellicle in semi-solid agar media. In this zone the concentration of dissolved oxygen permits optimal respiration rates without inhibiting nitrogen fixation (Day and Dobereiner, 1976).

As growth continues and more oxygen is consumed, the pellicle moves towards the surface where a dense pellicle forms. This growth pattern of Azospirillum in semi-solid media was reported by Hossain et al., (2015). Free living diazotroph, Azospirillum are able to survive even at $\mathrm{pH} 4.0$ i.e., under highly acidic conditions and they have wider availability in rhizospheric soils of different blocks of Ranchi district. They are negative to Gram's reaction.

They are vibroid shaped cells having capsule and are able to form thick walled microcysts during unfavourable conditions which is their adaptive mechanism to survive in adverse conditions. Azospirillum spp. show high degree of polymorphism in respect to their colonial patterns, elevation etc which may be attributed to their isolation from different rhizospheric and soil conditions where they were surviving. Colour development in few colonies is due to presence of carotenoid pigments.

\section{References}

Baldani, V.L.D., Alvarez, M.A., Baldani, J.I. and Dobereiner, J. (1986) Establishment of inoculated Azospirillum sp. in the rhizosphere and in roots of field-grown wheat and sorghum. Plant and Soil 90, $35-46$.

Becking, J.H. (1985) Pleomorphism in Azospirillum. In: Azospirillum III: Genetics, Physiology, Ecology. Edited by Klingmuller W. Springer, Berlin, pp 243262.

Beijerinck, M.W. (1925) Uberein spirillum, Welches freien stickstoff bidenkann. Central Baktparasit Infect II Abstract Edition 63, 353-359.

Berlman, J.E., Hasselbring, B.M. and Bauer, C.E. (2004) Hypercyst mutants in Rhodospirillum centenum identify regulatory loci involved in cyst cell differentiation. Journal of Bacteriology 186, 5834-5841.

Buchanan, R.E. and Gibbons, N.E. (1974) Bergey's Manual of Determinative Bacteriology, $8^{\text {th }}$ edition, Williams and Wilkins, Baltimore, pp. 196.

Day, J.M. and Dobereiner, J. (1976). Associative symbiosis in tropical grasses: characterization of microorganisms and dinitrogen fixing sites In: Proceedings of First International Symposium on $\mathrm{N}_{2}$ fixation (W.E Newton and C.J. Nyman Eds.), Washington University Press, Pullman W.A. pp 518-538.

Dobbelaere, S., Vanderleyden, J. and Okon, Y. (2003) Plant growth-promoting effects of diazotrophs in the rhizosphere. Critical Reviews in Plant Sciences 22, 107-149.

Gray, E.J. and Smith, D.L. (2005). Intracellular and extracellular PGPR: Commonalities and distinctions in the plant-bacterium signaling processes. Soil Biology and Biochemistry 37, 395-412.

Hartmann, A. and Zimmer, W. (1994) Physiology of Azospirillum. In: Okon Y. (ed.) Azospirillum-plant associations. CRC, Boca Raton, Fla. pp 15-39.

Hossain, M., Jahan, I., Akter, S., Md. Rahman, N. and Badier R.S.M. (2015) Isolation and identification of Azospirillum isolates from different paddy fields of North 
Bengal. Indian Journal of Research in Pharmacy and Biotechnology. 3, 74-80.

Jackson, M.L. (1973) Soil Chemical Analysis. Prentice Hall, New York, pp. 48-302.

Kreig, N.R. and Hylemon, P.B. (1976) Taxonomy of the chemoheterotrophic Spirilla. Annual Review of Microbiology 30, 303.

Lugtenberg, B.J.J., Chin-A-Woeng, T.F.C. and Bloemberg, G.V. (2002) Microbe-plant interactions: principles and mechanisms. Antonie van Leeuwenhoek International Journal of General and Molecular Microbiology 81, 373-383

Madi, L., Kessel, M., Sadovnik, E. and Henis, Y. (1988) Electron microscopic studies of aggregation and pellicle formation in Azospirillum sp. Plant and Soil 109, 115.

Magalhaes, F.M.M., Baldani, J.I., Souto, S.M., Kuykendall, J.R. and Doberiner, J. (1983) A new acid tolerant Azospirillum species. Annals of Academic Bras Cien 55, 417420.

Oh K.H., Seong C.S., Lee S.W., Kwon O.S. and Park Y.S. (1999) Isolation of psychrotrophic Azospirillum spp. and characterization of its extracellular protease. FEMS Microbiology Letters 174, 173-178.

Okon, Y. (1985) Azospirillum as a potential inoculant for agriculture. Trends in Biotechnology 3, 223-228.

Okon, Y., Albrecht, S.L. and Burris, R.H. (1977) Methods for growing Spirillum lipoferum and for counting it in pure culture and in association with plants. Applied and Environmental Microbiology 33, 85-87.

Rangaswami, G. and Bagyaraj, D.F. (1996) Agricultural Microbiology. Asian Publishing House, New Delhi.
Rasool, L., Asghar, M., Jamil, A. and Rehman, S.U. (2015) Identification of Azospirillum species from Wheat rhizosphere. Journal of Animal and Plant Sciences 25, 10181081.

Rosemary, O.C., OnahGloria, T. and Igbonekwu, C.C. (2013) Isolation and characterization of nitrogen-fixing bacteria in the soil. International Journal of Life Science and Pharma Research 2, 2250-3137.

Sachdev, D., Chaudhari, H.G., Kasture, V.M., Dhavale, D.D. and Chopade, B.A. (2009) Isolation and characterization of indole acetic acid (IAA) producing Klebsiella pneumoniae strains from rhizosphere of wheat (Triticum aestivum) and their effect on plant growth. Indian Journal of Experimental Biology 47, 993-1000.

Sadasivan, L. and Neyra, C.A. (1985) Flocculation in $A$. brasilense and $A$. lipoferum. Exopoly-saccharides and cyst formation. Journal of Bacteriology 163, 716.

Schroder, M. (1932) Die Assimilation des Luftstickstoffs durche lnige Bakterien. Zentbl Bakt. Parasitkde 85, 177-212.

Tarrand, J.J, Kreig, N.R. and Dobereiner, J. (1978) A taxonomic study of the Spirillum lipoferum group, with a descriptions of a new genus, Azospirillum gen. nov., and two species, Azospirillum lipoferum (Beijerink) comb. nov. and Azospirillum brasilense sp. nov. Canadian Journal of Microbiology 24, 967-980.

Yooshinan, S.U. (2001) Biological Nitrogen Fixation. Journal of Biological Association. 8, 50-55.

\section{How to cite this article:}

Narayan, R., N.C. Gupta and Shahi, D.K. 2018. Isolation, Morphological and Cultural Characterization of Azospirillum Isolated from Rhizospheric Soils of Various Non-Leguminous Crops of Ranchi Having Acidic pH. Int.J.Curr.Microbiol.App.Sci. 7(08): 329-338. doi: https://doi.org/10.20546/ijcmas.2018.708.037 